Our thanks are due to Mr. L. Phillips for analysing the gases from the thermal decomposition of nitroglycerin. This communication is published by permission of the Chief Scientist, Ministry of Supply.

Davy Faraday Laboratory A. J. B. ROBERTSON Royal Institution London, W.1.

\section{A. YoFFE}

Research Group on the Physics and Chemistry of Rubbing Solids,

Department of Physical Chemistry, University of Cambridge.

\section{Feb. 4.}

1 Robertson, R., and Garner, W. E., Proc. Roy. Soc., A, 103, 539 (1923). ${ }^{2}$ Haid, A., and Schmidt, A., $Z$. ges. Schiess- $u$. Sprengstoffw., 26, 253, 293 (1931).

${ }^{3}$ Robertson, R., J. Chem. Soc., 95, 1241 (1909).

${ }^{4}$ Appin, A., Chariton; J., and Todes, O., Acta Physiochim. U.R.S.S., 5, 655' (1936).

${ }^{5}$ Yoffe, A., Nature, [161, 349 (1948)]

- Taylor, J., and Hall, C. R. L., J. Phys. Coll. Chem., 51, 593 (1947)

' Bowden, F. P., and Gurton, O. A., Nature, [161, 348 (1948) ].

\section{'Semaphore' Numerals}

Enough is now known, as the Royal Society discussion on the subject on March 4 made clear [see Nature, May 8, p. 712], about the logical, physical and mechanical principles, and of the engineering, to make the existence of a number of high-speed digital computing machines a matter of time, and of comparatively short time, only. We can, in fact, do arithmetic at very high speed.

But the speed of any composite process is governed mainly by the speed of its slowest component. At present, 'input' and 'output' cannot take place at speeds in any way comparable with the attainable speed of computation. The machine should be able to do all three of the 'three R's' at comparable speedsit must learn to Read and to wRite, and to do these quickly, as well as to do aRithmetic! Reading is the most important, for it should occur at a rate per digit comparable with an 'addition time'; but 'output' will not slow down the process if recording a result takes no longer than the chain of arithmetical operations which produces it.

Towards the end of the recent discussion, Mr. Wilkinson remarked that he had given some thought to the processes by which the human eye recognizes the numerals as printed or written, and seemed to imply that present forms would prove difficult for the lowgrade intelligence of the robot to recognize. The alternative is, presumably, to introduce a new, simple and systematic set of numerals, simple enough for the robot but usable also by the human, so that mathematical tables might be printed in a form universally available, needing no further coding for the robot. We present here a possibility in this direction. While it would be unwarrantable optimism to presume that it is the last word, it is upon some such lines that progress depends, and a concrete suggestion may stimulate thought and discussion which vague generalities would not.

Working in the binary scale would be too cumber. some; working in some power of 2 , say, 8 or 16 , would not be too remote from the decimal scalebut it would entail the re-learning of arithmetic, 'multiplication tables and all that'. Indeed, a proposal to abandon the decimal system would be too revolutionary to stand any chance of general accept- ance. But, fortunately, $10-1=3^{2}$, so that a dual use of a trinity of symbols will suffice. Such a possibility is presented by the three positions of a semaphore, upright, or inclined to left or right. The combination of upward-pointing and downwardpointing triads gives us the nine digits. A scheme, easy to write, easy to read-and very easy to remember-is presented in Fig. 1.
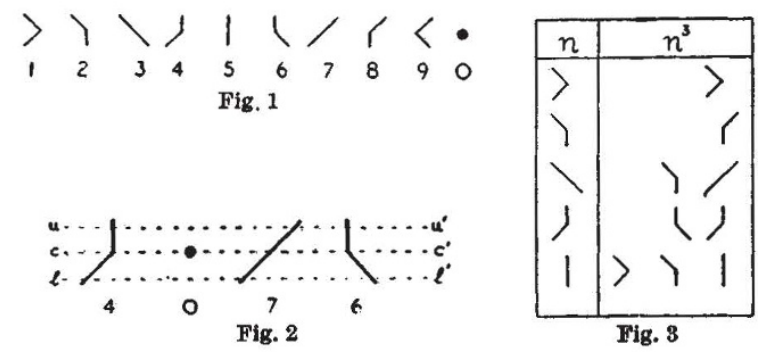

The provision of a zero can be met by a central dot (a mere blank is not satisfactory). In writing, the comma can (as it now does in many countries) serve as a decimal point, and a lower point couldif desired-be used to indicate multiplication, but these would not be relevant to 'reading' by the robot.

The machine 'scanning' of the new numerals could be horizontally, for serial operation. Two 'eyes' are essential, but a third central one is desirable, to provide a time-scale and to sense the zeros.

The method is indicated by the dotted lines in Fig. 2. The three eyes will scan the lines $u u^{\prime}, c c^{\prime}$, and $l^{\prime}$, and the impulses due to crossing the lines or the dot will be interpreted by circuits in the machine, so that the number read is fed into the appropriate register.

If this, or some similar, suggestion is accepted, it remains to fix an agreed standard size and spacing, and to use these numerals in printing all new mathematical tables. Re-coding of existing tables could also be undertaken. Some idea of appearance is given in Fig. 3, representing the beginning of a table of cubes.

Visual users of mathematical tables could rapidly master (and indeed use) the new code, and the problem of making robots wRite would be in no way more difficult.

\section{Imperial College, \\ London, S.W.7. March 9. \\ Angular Distribution of Radioactive Disintegration Products}

THE thermodynamics of the orientation of nuclei by strong magnetic fields at very low temperatures was worked out by Simon ${ }^{1}$ in 1939 . He found that with fields of 100,000 gauss and temperatures of $0.01^{\circ} \mathrm{K}$., that is, under conditions which can be realized experimentally, the entropy of nuclear spin systems should be decreased by about 20-30 per cent, and would remain, of course, at this reduced value after adiabatic demagnetization. This would be sufficient to influence properties which depend on nuclear orientation, and the first experiments he contemplated were on the angular distribution of radioactive disintegration products originating from a partially oriented nuclear spin system. Prepara- 